\title{
Current perspectives in robotic hernia repair
}

This article was published in the following Dove Press journal:

Robotic Surgery: Research and Reviews

5 May 2017

Number of times this article has been viewed

\section{Charan Donkor ${ }^{1,2}$ \\ Anthony Gonzalez ${ }^{1,2}$ \\ Michelle R Gallas ${ }^{3}$ \\ Michael Helbig ${ }^{2}$ \\ Corey Weinstein ${ }^{2}$ \\ Jaime Rodriguez ${ }^{2}$}

'Department of General and Bariatric Surgery, Baptist Health South Florida, Miami, FL, USA; ${ }^{2}$ Florida International University, Herbert Wertheim

College of Medicine, Miami, FL, USA;

${ }^{3}$ Population Health and Outcomes

Research, Center for Research and

Grants, Baptist Health South Florida,

Miami, FL, USA
Correspondence: Charan Donkor Department of General and Bariatric Surgery, Baptist Health South Florida, 7800 SW 87th Avenue, Suite B2I0,

Miami, FL 33173, USA

Tel +l 305 27| 9777

Email charand@baptisthealth.net
Abstract: The surgical treatment of hernias has developed throughout the evolution of surgery. The fascination with hernia surgery is in part driven by its prevalence and by the variety of treatment options. Minimally invasive hernia surgery has a goal of a robust repair with minimal complications, and new robotic techniques are being developed in complex abdominal wall hernias with promising results. This review focuses on inguinal, ventral, and incisional hernias and their outcomes with a discussion on the traditional open, laparoscopic, and robotic techniques. The prevalence of minimally invasive hernia surgery and its advantages are also outlined. We highlight our experience in these procedures, specifically robotic herniorrhaphy, as it pertains to ventral incisional and inguinal hernia repair. We conclude that the robotic platform is proving to be a benefit to hernia repair. Many studies are showing its feasibility and comparable results to standard laparoscopy, and some have shown improved results, including shorter hospital stay without significant increases in cost. The robotic option of hernia repair has resulted in an increase in minimally invasive hernia repair, a number that has remained stagnant for the last decade. With more surgeons gaining training and experience and greater availability of the robotic platform, we expect to see greater numbers of minimally invasive hernia repair.

Keywords: hernia, hernia repair, ventral, incisional, robotic, laparoscopic

\section{Introduction}

\section{Hernia overview}

The concept of hernias and their treatment have been present since the beginning of human history. ${ }^{1}$ Treatment of hernias has progressed alongside the development of surgery, and the evolution of hernia repair has been facilitated by new prosthesis technology. The fascination with hernia surgery is in part driven by its prevalence and by the variety of treatment options. Herniorrhaphy, however, is not without its risks of complications. The goal of hernia surgery is for a robust repair while minimizing complications. Minimally invasive hernia surgery treats the problem with less risk for common complications encountered with open surgery. New techniques in minimally invasive hernia surgery are arising from previous open methods, and more complex abdominal wall hernias are now approached robotically. These results are promising for patients with complex abdominal hernias who may require a strong anatomic repair but who can still benefit from a minimally invasive approach. An abdominal wall hernia is defined as the protrusion of intra-abdominal tissue through a defect in the abdominal wall, known as ventral hernia. Hernias can be either the enlargement of a natural anatomic defect (hiatal or inguinal hernias) or the development of a new defect in a compromised tissue. For the purpose of this discussion, we will concentrate 
on inguinal, ventral, and incisional hernias. The ventral hernia category includes umbilical, epigastric, and spigelian hernias.

In this review, we discuss traditional open and laparoscopic hernia repair and outcomes. The prevalence of minimally invasive hernia surgery and its advantages are outlined. We highlight our experience in minimally invasive hernia surgery, specifically robotic herniorrhaphy, as it pertains to ventral incisional and inguinal hernia repair.

\section{Hernia surgery}

Open hernia repair is considered as a "bread and butter" operation for the general surgeon, and therefore its management is of great significance. Although hernias are some of the most common pathologies seen by physicians, their treatment can vary widely among surgeons due to a lack of consensus in management. ${ }^{2}$ Hernias are repaired via traditional open surgery or by a minimally invasive technique. Minimally invasive hernia surgery is associated with similar recurrence rates and less complications such as surgical site infections. ${ }^{3}$ Despite these advantages afforded by minimally invasive surgery, traditional open hernia surgery still seems to be the mainstay of treatment as the majority of abdominal wall hernias are repaired in this manner. With that being said, the majority of ventral and inguinal hernias are still repaired with an open technique., ${ }^{3,4}$ Due to the degree of technical difficulty, the learning curve for minimally invasive hernia repair is steep, and this is a major factor contributing to this low number. With the advent of the robotic platform, this number is increasing. The da Vinci robotic platform allows surgeons to complete technically difficult surgeries minimally invasively because of better visualization and the capability of wristed movements. Robotic surgery is now also playing an important role in complex abdominal wall hernias. Open complex hernia surgery has a significant complication rate, and not much has changed over the past 20 years in surgical technique. Alongside the development of technique is the evolving material bioscience of mesh prosthesis, and these two go hand in hand when discussing current trends in robotic-assisted hernia surgery.

\section{Mesh selection}

Another component of this highly debatable topic is mesh selection. Other than biologics, polypropylene and polyester are the most common materials used to manufacture mesh prostheses. The manufacturing process begins from raw material that is processed into polypropylene or polyester pellets. These are extruded into fibers and are then woven into a particular pattern that has been preset. This pattern is important for the characteristics of the final products. An ideally engineered prosthesis should be strong, flexible, nonallergenic, and noncarcinogenic and stimulate fibroblast activity. Elasticity, stiffness, burst strength, tensile strength, and compliance are the parameters that are heavily studied in detail when developing a prosthesis. These can be related to the density, weight, and pore size. The mesh can also be coated with different materials if needed for purposes of intra-abdominal placement. Therefore, there are numerous varieties that are possible and few studies with strong evidence indicating the best mesh prosthesis. Many surgeons may not be aware of their slight differences, and their preferences may be based on other factors such as availability and cost. Hospitals for example may provide contracted products with certain companies exclusively. Decisions as to which products are available are often made by either nonclinical administrators or a small group of surgeons in that hospital. This typically keeps cost at a minimum but limits the surgeon's options and therefore experience with other products.

Pore size, density, and tensile strength are important properties when selecting a mesh. ${ }^{5}$ There is good evidence that use of a lightweight mesh is not appropriate for a "bridging" repair of a ventral hernia, and lightweight mesh may also not be appropriate for inguinal hernias with a large direct defect. Adhesion formation is also an important factor especially when dealing with subsequent abdominal operations. ${ }^{6}$ There exists ample research in adhesion barriers; however, the evidence is not as clear as to which barrier is best. All of these points discussed earlier should be taken into account when selecting a mesh. In addition, surgeons should be comfortable, familiar, and confident in the product that they decide to place within their patient's abdominal cavity.

\section{Mesh fixation}

The increase in the use of mesh in the last 20 years has helped to reduce the rates of recurrence. The goal of mesh fixation is to allow adequate adhesion of the mesh while it is allowed to incorporate into the tissues of the abdominal wall with minimal postoperative pain. The outcome is preventing a recurrence of the hernia and avoiding chronic pain. Tackers, transfixation sutures, glue, and self-adhering mesh are the options currently available to the surgeon. There has been little standardization of mesh fixation due to the variety of methods available, which is due in part to the lack of strong evidence coupled with the fact that hernia technology is constantly changing. Pain has been associated with tackers and transfascial fixation sutures, and so there has been motivation to create adherence in 
other ways. There are concerns among surgeons that the use of tack mesh fixation (TMF) for laparoscopic inguinal hernia repair carries with it a significant risk of pubic osteitis, nerve entrapment, and chronic groin pain. ${ }^{7}$ A metaanalysis of TMF vs glue mesh fixation (GMF) published in 2013 found that there was no difference in the rates of operative time, perioperative pain, postoperative complications, recurrence, and length of stay. There was, however, a difference in the development of chronic groin pain, with those in the GMF group having a significantly lower rate of chronic pain. In this comparison, however, only four studies contributed to the analysis, and these studies had moderate to substantial heterogeneity. ${ }^{7}$ Overall, it seems that use of glue may have comparable results to fixation with tackers when applied in inguinal hernia repairs. ${ }^{8}{ }^{8}$ In ventral hernias, transfixation sutures and a double crown technique of tackers seem to carry the most security for mesh adherence. ${ }^{10}$ In robotic ventral hernia repair, mesh can be fixed by either of these ways or even with suturing in a running or interrupted fashion with comparable results. ${ }^{11}$ Self-adhering mesh has been described in inguinal hernias with or without added fixation, and preliminary results are encouraging. ${ }^{12}$ Similar to mesh selection, fixation choice is ultimately up to the surgeons and dependent on their personal experience. As more studies are published, standardization may be attained.

\section{Open inguinal hernia repair}

Open inguinal hernia repair remains the mainstay of inguinal hernia repair, with the majority of elective inguinal hernia repairs being done via the traditional open technique. The modern treatment of groin hernias began with primary repair under tension. Bassini is well known for his anatomical repair of the posterior wall of the inguinal canal. ${ }^{13} \mathrm{He}$ collected his data on $>200$ patients and followed their surgical outcomes. This was followed by Lichtenstein and Shulman ${ }^{1}$ who described the most popular technique to date; tension-free repair with mesh. In his technique, a plug is placed within the external ring and a patch over the inguinal floor. The mesh is then sutured in place to the conjoint tendon, shelving edge, and pubic tubercle. Lichtenstein and Shulman ${ }^{1}$ popularized this technique by publishing their first 1000 cases with excellent results. This revolutionized hernia repair, and as a result recurrence rates dropped. Although a variety of mesh prostheses exist, the actual technique has not changed significantly. Complications include wound infection, postoperative pain, and hernia recurrence. When compared with minimally invasive hernia repair, wound infections are decreased and postoperative pain and recurrences tend to have similar rates as their open counterpart. ${ }^{3}$

\section{Laparoscopic inguinal hernia repair}

Laparoscopic repair of inguinal hernia was first described in 1979 where he closed the neck of the sac laparoscopically. ${ }^{2}$ The technique now most commonly practiced is categorized as either transabdominal preperitoneal (TAPP) approach or totally extraperitoneal (TEP) approach and involves the placement of a mesh. Both techniques have similar outcomes in operative time, postoperative pain, return to work, and recurrences. ${ }^{14}$ There are some reported advantages of performing TAPP in patients with contralateral hernias and incarcerated hernias where direct visualization of the bowel is important. When compared with open hernia repair, laparoscopic repair leads to less postoperative pain and wound infections and earlier return to work. ${ }^{3}$

Despite these advantages in laparoscopic inguinal hernia repair, adoption into standard practice has been in stark contrast to laparoscopic cholecystectomy that has become the standard of care since the first case was performed in $1985 .^{15}$ This is due to the steep learning curve even for those who have completed a fellowship in minimally invasive surgery. Despite this advanced training, not all fellowship-trained surgeons perform minimally invasive inguinal hernia repair in their practice. Part of the difficulty arises from technical aspects, such as a smaller working space and difficulty in handling the mesh laparoscopically. In addition, the anatomic considerations differ because the landmark structures are visualized from a different vantage point. It may take $\sim 20$ cases in order to become familiar with this anatomy and stabilize operating times. ${ }^{16}$ Laparoscopic repair, however, should be the choice for bilateral inguinal hernias and recurrent hernias and is acceptable but debatable for unilateral hernia repair.

\section{Robotic inguinal hernia repair}

Due to the fact that laparoscopic inguinal hernia repair is not widely practiced, the robotic platform has become an integral part in minimally invasive inguinal hernia repair. Wristed movements, three-dimensional visualization, and excellent ergonomics for the surgeon have contributed to this technique becoming a more adoptable technique than its laparoscopic counterpart. The first reports of robotic inguinal hernia repair were reported by urologists while performing radical prostatectomy. ${ }^{17,18}$ Since these initial reports, publications by general surgeons of formal robotic inguinal hernia repair have become available. Escobar Dominguez et $\mathrm{al}^{19}$ 
published his series of 78 patients who underwent robotic inguinal hernia repair and showed comparable results with similar cost implications. Waite et $\mathrm{al}^{20}$ published a smaller series of robotic TAPP vs laparoscopic TAPP and showed improved postoperative pain with an increase in operating time and similar cost.

Cost containment is a valid concern for all those caring for patients. On initial impression, one may be led to believe that robotic hernia repair is cost prohibitive. On further inspection and in most cases, the robot has already been purchased for specialties such as urology and gynecology. Compared to its laparoscopic counterpart, the surgeon can significantly cut the cost of the procedure by eliminating a balloon spacer for those practicing standard laparoscopic TEP. For surgeons who use a tacker device, suturing or self-adhering mesh are reasonable alternatives. In the robotic repair, TAPP is more commonly performed because in this technique, there is a greater working space, which makes the procedure technically easier. This point is accentuated in patients with a smaller body habitus. Overall, we can expect to see an increase in surgeons performing minimally invasive hernia surgery because of the technical ease of the robotic platform. This translates into a patient benefit, since minimally invasive hernia repair has multiple benefits both short and long term.

\section{Surgical technique - robotic inguinal hernia repair}

Robotic platforms Si and Xi are optimal for robotic inguinal hernia repair. When using the Si platform, location of cart placement for docking is paramount. Conversely, the $\mathrm{Xi}$ platform allows side docking in all cases. Entry into the abdominal cavity is gained via a periumbilical incision. If the patient has a previous midline incision and entry at the midline is not safe, entry at Palmers point (primary left upper quadrant) is used instead. Access to the peritoneal space is accomplished with placement of a Veress needle, optical trocar, or direct cut down using the Hassan technique as per the surgeon's choice. If a Hassan technique is used, it needs to be with a trocar that is compatible with the robotic platform being used. Insufflation with $\mathrm{CO}_{2}$ to $15 \mathrm{mmHg}$ is standard. The remaining bilateral robotic trocars are introduced under direct laparoscopic vision. These are generally placed at the level of the umbilicus or slightly higher depending on the abdominal girth of the patient. For patients with smaller body habitus, we place our trocars more cephalad to ensure adequate working space (Figure 1). On the da Vinci Si platform, docking the robotic cart can be accomplished between the legs with the patient in lithotomy or laterally with the patient supine (Figure 2). Our position of choice is supine with moderate Trendelenburg. Docking does not need to be performed ipsilateral to the hernia to be repaired. Parallel or side docking will afford access to both inguinal regions irrespective of the location of the robotic cart (Figure 2).

The Xi model allows docking from either side. TAPP remains the approach of choice for the robotic inguinal hernia repair. The anatomy differs in this approach from the traditional open approach. Prior to opening the peritoneum, the median umbilical ligament, external iliac vessels, and gonadal vessels should be identified. We begin with a peritoneal incision between the anterior superior iliac spine and the medial umbilical ligament. Opening of the peritoneum and dissection can be performed by scissors or hook with cautery. The vessel sealer device for the robot may be utilized for this purpose as well but is more costly. Consideration of the cost of instrumentation must be recognized, so we make an effort to limit our instrument use to a combination of three
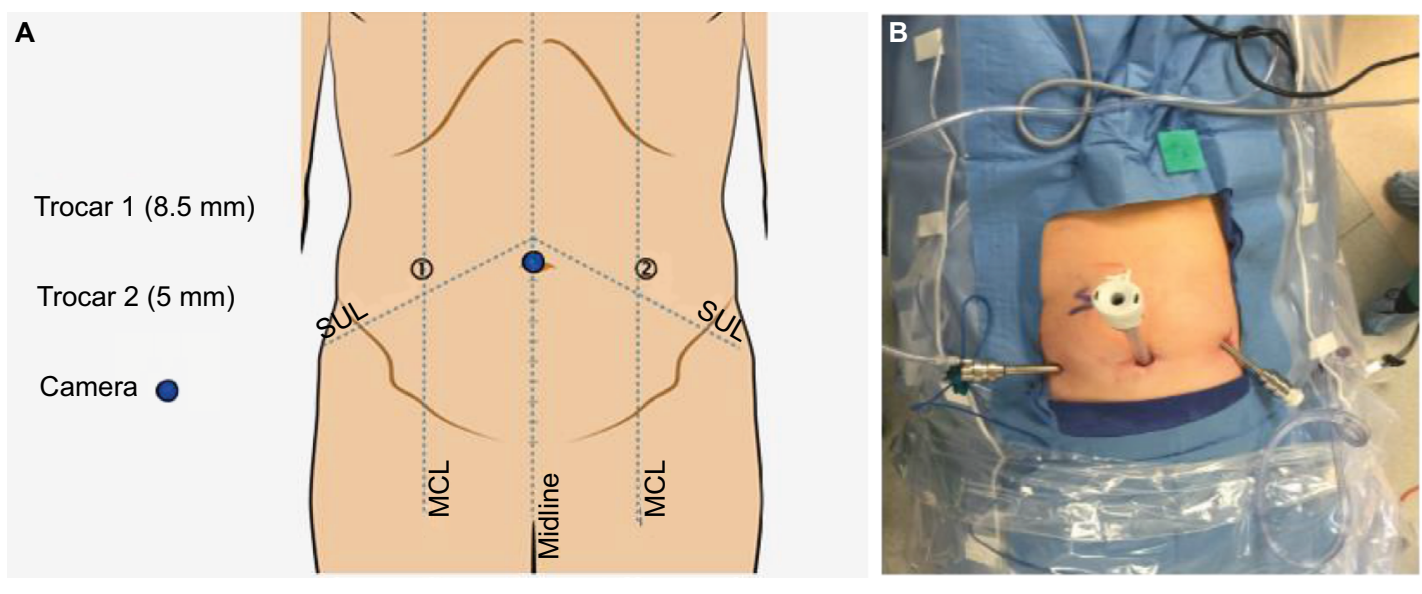

Figure I Schematic representation of trocar placement illustrating cephalad orientation (A) and example of actual cephald trocar orientation (B). Abbreviations: MCL, midclavicular line; SUL, spinoumbilical line. 


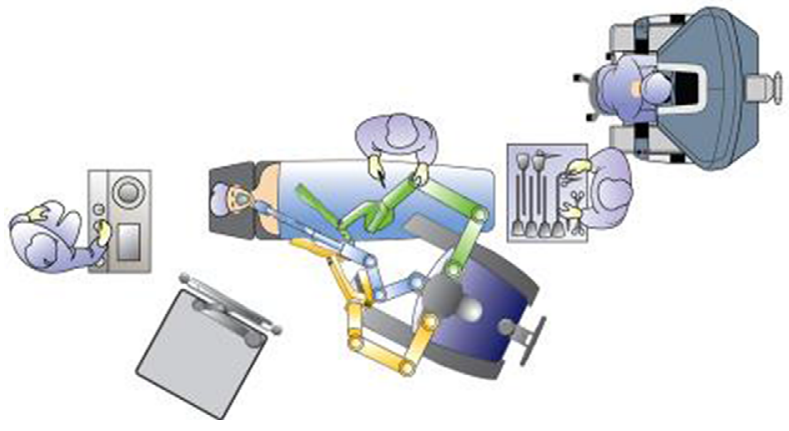

Figure 2 Robot side docking with da Vinci Si allows access to bilateral inguinal regions.

instruments. For example, a typical robotic inguinal hernia may use a grasper, scissors with cautery, and a suture cut needle driver. The incision must be wide enough to access to Cooper's ligament and the lateral abdominal wall and also large enough to incorporate an appropriate sized mesh comfortably. The flap size is generally estimated to fit the mesh snugly. Too large a flap will add operative time and allow easier mesh migration, whereas a flap and dissection that is too small will not allow comfortable placement of the appropriate sized mesh. In the latter scenario, the mesh may roll up at the inferior margin and this can lead to a recurrence.

In our technique, once our flap is created, we focus on dissecting Cooper's ligament just lateral to the symphysis pubis. This will help in recognizing the preperitoneal anatomy. Cooper's ligament is identified first by dissection of the medial space. This is done typically before addressing the hernia as it serves as a useful landmark especially when the hernia sac is large and obscuring the field of dissection. Once Cooper's ligament is identified and is in clear view, we encourage a lateral dissection of the preperitoneal space. This will ensure an easier identification of the spermatic cord, vas deferens, and other important structures (Figure 3). The hernia sac must be detached completely from the spermatic cord to avoid hernia recurrence. In cases of large inguinoscrotal hernias in which dissection may become challenging, the sac may be transected and left in place being careful to close the peritoneal defect. If the decision has been made to ligate the sac, the narrowest point of the sac is optimal, as this will reduce the size of the defect in the peritoneal flap requiring closure. Once the dissection is complete, the mesh can be placed into proper position. Delivery of the mesh can be at the beginning of the procedure prior to docking the robot or at the appropriate time by the assistant at the bedside via an $8 \mathrm{~mm}$ robotic trocar. When placed at the beginning of the procedure, we

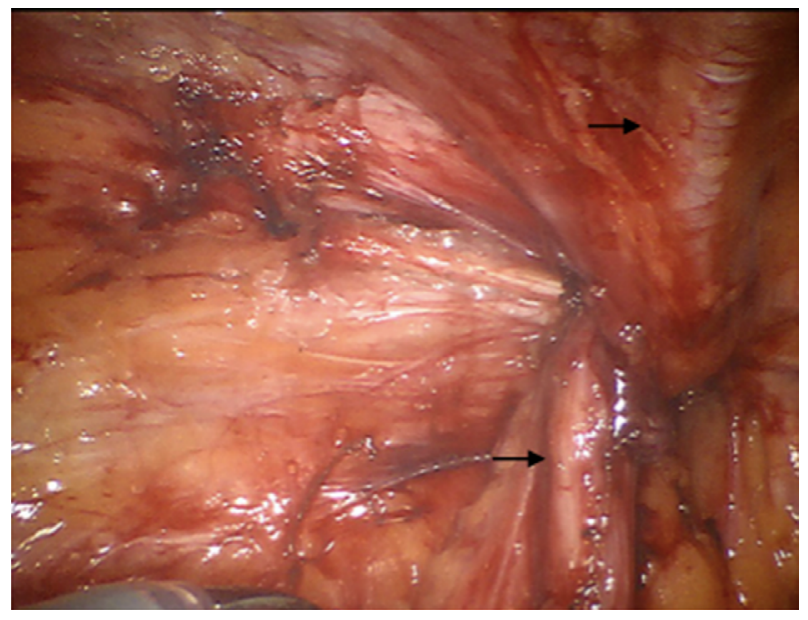

Figure 3 Illustration of inguinal dissection.

Notes: Top arrow shows the epigastric vessels, and the bottom arrow shows the vas deferens within the spermatic cord.

place our mesh and suture in the pelvic inlet where it is in constant view but out of the way of the dissection. When using a self-adhering mesh, suture fixation is needed when the direct defect is large. The mesh spans the space from the pubic tubercle to the lateral abdominal wall covering both indirect and direct defects. If fixation is to be performed, nonabsorbable sutures or tackers are placed at Cooper's ligament and the upper abdominal wall both medial and lateral to epigastric vessels. Self-adhesive mesh must be carefully and strategically placed to prevent the need to "un-attach" the mesh and reapply. We prefer to roll the mesh from medial to lateral starting at Cooper's ligament. This ensures that there is sufficient coverage of the medial space when positioning. Adequate coverage is key as this can lead to reduction in recurrences (Figure 4). In addition to this technique, a large direct defect can be closed with barbed suture. This may serve to prevent eventration of the mesh through large defects.

The peritoneal flap is then closed with a running barbed absorbable or permanent suture. The use of barbed suture has facilitated this closure since it maintains the "tension" during reapproximation. There have been some case reports of bowel obstruction after surgery due to adherence of the barbed suture to the intestine. ${ }^{21}$ Since exposed long segments of suture can allow bowel to be entrapped, we make an effort to "cinch" down the suture as much as possible in order to bury it within the peritoneum (Figure 5). Overall, barbed suture is considered to be safe in intra-abdominal surgery; however, the surgeon should be aware of this uncommon complication. 


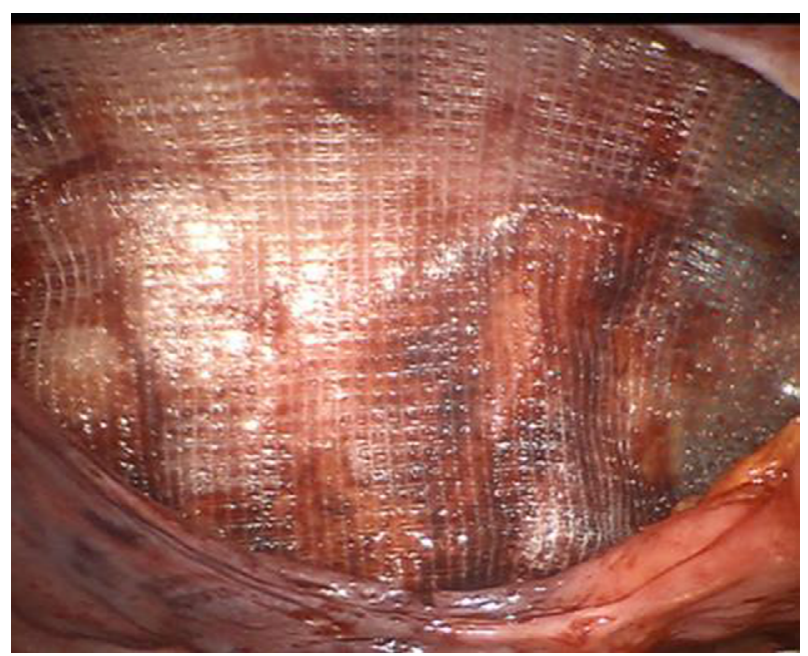

Figure 4 Once the hernia sac has been reduced, the mesh is placed in proper orientation with adequate coverage of direct and indirect space.

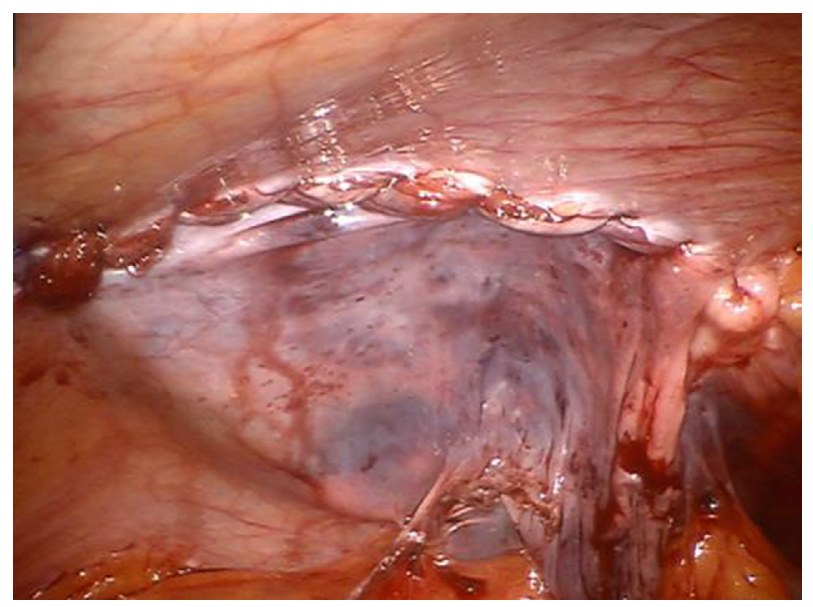

Figure 5 Burying the barbed sutures within the peritoneum ensures adequate closure and protects against complications such as bowel entrapment.

\section{Ventral and anterior incisional abdominal wall hernias}

Anterior incisional and ventral abdominal wall hernias are a common pathology. Incisional hernias develop after a previous surgery, and this occurs approximately at a rate of $15-20 \%$. It is estimated that $\sim 400,000-600,000$ incisional hernias are repaired each year in the USA. ${ }^{22,23}$ The risk of developing an incisional hernia increases yearly from the time of surgery. The treatment is surgery, and traditionally these hernias are repaired as an open procedure with or without the placement of mesh prosthesis. There are a variety of ways to repair these hernias as well as a large variety of prostheses to choose from. The surgeons ultimately decide which technique will best suit their patient. A variety of techniques to repair hernias exist. Due to this, there is a lack of standardization of the surgical treatment; therefore, debate exists when discussing optimal technique, patient selection, and choice of

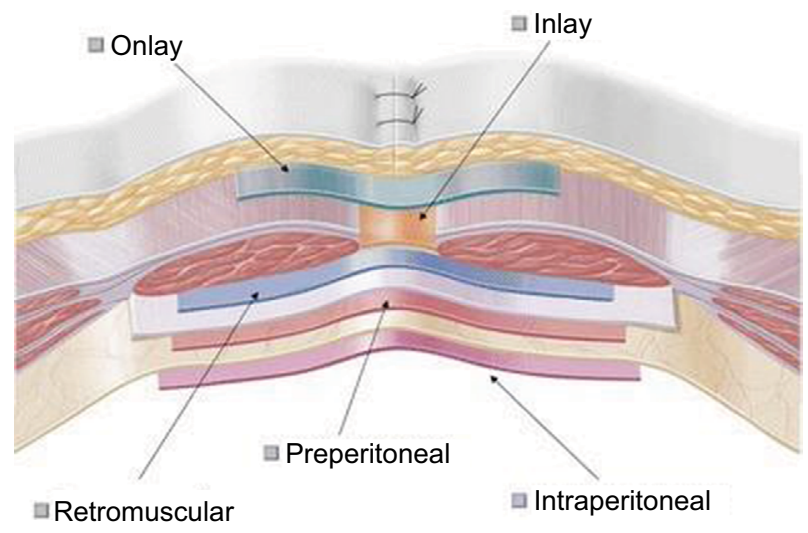

Figure 6 Outline of different anatomical positions for mesh placement. Note: (C) The Author(s) 2012.30

prosthesis used. Research on this subject comes from pooled data, and therefore interpretation can be confusing.

The mesh during repair can be placed in different positions anatomically, and this is consistently a topic of debate (Figure 6). Mesh that is placed above the fascia is referred to as "onlay". Mesh that is placed between the posterior rectus sheath and rectus muscle refers to a sublay placement. Finally, when the mesh is placed against the peritoneum, it is considered to be in an underlay position. A large Cochrane review ${ }^{24}$ compared onlay vs sublay mesh placement and failed to show an advantage with either technique. Mesh repair was associated with less recurrences, and a standard weight mesh showed a lower recurrence weight than the lightweight mesh. ${ }^{24} \mathrm{We}$ do know that for large complex abdominal wall hernias, morbidity is high and, if they can be repaired with a minimally invasive approach, risk to the patient can be significantly decreased. Patient selection is key for this approach, since multiple previous abdominal surgeries, previous mesh placement, and large size hernia defects may be relative contraindications to the minimally invasive approach. Larger defects may show better outcomes with an open technique depending on the surgeon's experience. In patients with large complicated abdominal wall hernias, a primarily closed defect may be optimal for long-term results. ${ }^{25}$ With the advent of the robot, this is now being done minimally invasively and the results are promising. The morbidity of a large open incision is removed, and the strength of the hernia repair is preserved. In order to illustrate this clearly, we begin by discussing open ventral and incisional hernia repairs.

\section{Open ventral hernia repair (OVHR) with or without mesh}

The traditional open hernia repair is described with or without the use of mesh. It has been shown that mesh placement decreases the recurrence rate; therefore, in cases 
where mesh is appropriate, a prosthesis should be used. ${ }^{24}$ There are different locations as to where to place the mesh, and this adds to the variety of techniques. Onlay refers to placing the mesh directly on top of the fascia just under the subcutaneous tissue. Although this is not the most popular, it has been shown that this placement is just as effective as others. ${ }^{26}$ The underlay mesh is placed against the peritoneum and is optimal when $4-5 \mathrm{~cm}$ of overlap is achieved. The underlay placement is most typically performed by circumferentially suturing the mesh to the underside of the fascia against the peritoneum while achieving 4-5 cm overlap. The fascia is then typically closed on top of the mesh. This can be done with or without the use of a component separation. Rives et $\mathrm{al}^{27}$ described the sublay technique where the mesh is placed retromuscularly. This can be combined with a component separation also if necessary to approximate the fascial edges. Whether the position of the mesh impacts outcome is a topic of debate: sublay has been shown to be superior to underlay with respect to recurrences. ${ }^{28}$ However, sublay compared to onlay has also been claimed to show no advantage to the sublay technique, while others have shown significant advantage. ${ }^{28,29}$ The debate continues, and trends toward underlay repair are prevalent in the USA, whereas in Europe, sublay repair is standard.

\section{Laparoscopic ventral and incisional hernia repair (LVIHR) with mesh}

Laparoscopic ventral hernia repair was originally described in the early 1990 s as a bridging intraperitoneal onlay mesh (IPOM) repair. Metallic tacks were originally used to secure the mesh, and later transfascial fixation sutures were implemented, which reduced the recurrence rate. ${ }^{30}$ LVIHR is most commonly performed as a laparoscopic IPOM repair. The described technique is quite standard and consists of placing mesh prosthesis to "bridge" the hernia defect. Important evidence-based technical points include mesh overlap, use of transfascial fixation sutures, and "double crown" technique for tacker placement. Shorter length of stay, quicker return to work, and significantly lower infection rates when compared with open hernia repair are proven benefits of LVIHR. ${ }^{31,32}$ Another benefit of laparoscopy is the ability to evaluate the entire fascia, and therefore concurrent hernias can be detected and repaired at the same time. Even though morbidity is decreased, still only $20-27 \%$ of ventral hernias are repaired laparoscopically. ${ }^{4}$ Typically, in LVIHR, the fascial defect is not closed. Larger defects tend to have higher recurrence rates with this "bridged repair". Drawbacks to LVIHR include eventration of the mesh, and more acute and chronic pain. ${ }^{33} \mathrm{In}$ addition, the placement of an intraperitoneal mesh increases the risk of enterotomy in subsequent abdominal surgeries. ${ }^{34}$ It is thought that the bridged repair is less robust because the forces exerted by the oblique muscles on the mesh can lead to recurrence. In addition, when the fascia is approximated in the midline, the forces are more evenly distributed and therefore the repair is stronger. It has been shown that an improved success of hernia repair when closing the defect along with mesh placement is achieved. ${ }^{25}$ This is done infrequently laparoscopically and only by the most skilled of minimally invasive surgeons. Therefore, the benefits of LVIHR with the benefits of traditional open technique may lead us to better results in these patients. This has not been done routinely until the recent utilization of robotics in hernia repair.

\section{Robotic ventral hernia repair}

With the advent of robotics, larger and more complex hernia repairs are being approached in a minimally invasive fashion. Benefits of fascial closure, retrorectus placement of the mesh, rectus muscle release, and intraperitoneal suturing of the mesh are facilitated by the minimally invasive robotic platform. The goal of robotic hernia repair is to obtain the same quality of hernia repair that has been achieved through the open technique while eliminating its perioperative morbidity. Although fascia closure and position of the mesh are debatable, robotic-assisted surgery has given the minimally invasive hernia surgeon more options than may have been available with standard laparoscopy. IPOM and IPOM-plus (ie, IPOM with intracorporeal fascial closure) have typically been the two options for the minimally invasive hernia surgeon. Allison et $\mathrm{a}^{35}$ studied the robotic laparoscopic incisional hernia repair with intracorporeal fascial closure. This study looked at 13 patients with small and medium sized ventral hernias with an average fascial defect of $37.39 \mathrm{~cm}^{2}$. The defect was closed using a running $\mathrm{O}$-absorbable suture. Average operating time was 131 minutes, with a morbidity rate of $13 \%$. The mean hospital stay was 2.4 days, and only one patient experienced a recurrent hernia. Although this was a small study with a short follow-up of 23 months, it showed the feasibility of robotic-assisted hernia repair with comparable results. ${ }^{35}$ Gonzalez et al $^{11}$ described robotic ventral hernia repair with fascial closure and compared this with a laparoscopic cohort. In this method, the fascia was closed robotically without excising the sac. An intraperitoneal mesh was then sutured into place in the intraperitoneal underlay position. Safety and efficacy was evident in this repair. Of note, were the lower recurrence rates in the robotic group who underwent fascial closure. Early studies in robotic-assisted hernia repairs have 
generally approached small-to-moderate size hernias. Warren et $\mathrm{al}^{36}$ described his retromuscular and transabdominal rectus (TAR) release repair and compared it with the conventional laparoscopic repair. Hernias were typically larger with a mean area of $88 \mathrm{~cm}^{2}$. In this technique, the posterior fascia is incised to the semilunar line and the rectus muscle is then released if necessary. Closure of the defect is achieved even for large defects, and the mesh is excluded from the intra-abdominal compartment by placing it retrorectus and preperitoneal. At least $5 \mathrm{~cm}$ of overlap is the goal when repairing the hernia. In this single institution study, 103 patients who underwent laparoscopic ventral hernia repair were compared with 53 patients who underwent robotic retromuscular ventral hernia repairs. Having adjusted for age, demographics, and hernia, it was found that fascial closure was achieved $96.2 \%$ of the time with the robot while only $50.5 \%$ of the time with the laparoscopic technique. Operative times, however, were considerably longer with the robot with 245 minutes as the mean operative time vs 122 minutes. The robotic technique fared better than laparoscopic in postsurgical narcotic requirements with 0.4 less morphine equivalents required as well as 1 whole day less for a median length of stay. Conversely, seroma occurred at a higher rate in the robotic cohort with 47.2 vs $16.5 \% .{ }^{36}$ Liang et $\mathrm{al}^{37}$ published a systemic review of best practice for ventral hernia repair. Expert panel responses were used to develop a consensus where all experts agreed. Fascial closure and a minimally invasive technique have evidence-based benefits, and therefore, robotics help to facilitate this. Debate lies in whether the sac should be excised and also in the position of the mesh. Some have mentioned to incorporate the sac within the closure of the fascia in order to decrease seroma formation; however, there are no data to support this to date. ${ }^{36}$ With regard to positioning of the mesh, the data are variable with some studies showing a sublay position to be best. ${ }^{37}$ Ultimately, this decision is up to the surgeon performing the surgery. If the surgeon feels that a sublay position would be better for a particular patient, a robotic retrorectus repair is an option.

\section{Surgical technique: robotic ventral incisional hernia repair}

In robotic ventral hernia repair, abdominal access is obtained as previously described. Ports are placed quite lateral in order to allow as much working space as possible (Figure 7). In very large hernias, a double docking technique may be necessary. Therefore, additional ports can be placed in the same positions on the opposite side (Figure 8). Any adhesions that will prevent the dissection are taken down laparoscopically. We start the retrorectus dissection by incising the contralateral posterior rectus sheath. We typically use the hot shears for this part of the dissection. In cases where the defect is off midline, we can enter the retrorectus space medially and work our way laterally. For central hernias, we typically begin just off the midline and incise the posterior rectus sheath (Figure 9). Lateral dissection is carried out on both sides by the double docking technique. Dissection $5 \mathrm{~cm}$ superior and inferior to the borders of the hernia defect allows adequate placement of an appropriately sized mesh. Mesh selection is up to the surgeon. The lateral boundary is the lateral perforating vessels, and the dissection is carried just medial to these. The sac is excised if possible (Figure 10). In some cases, we have chosen to leave the sac
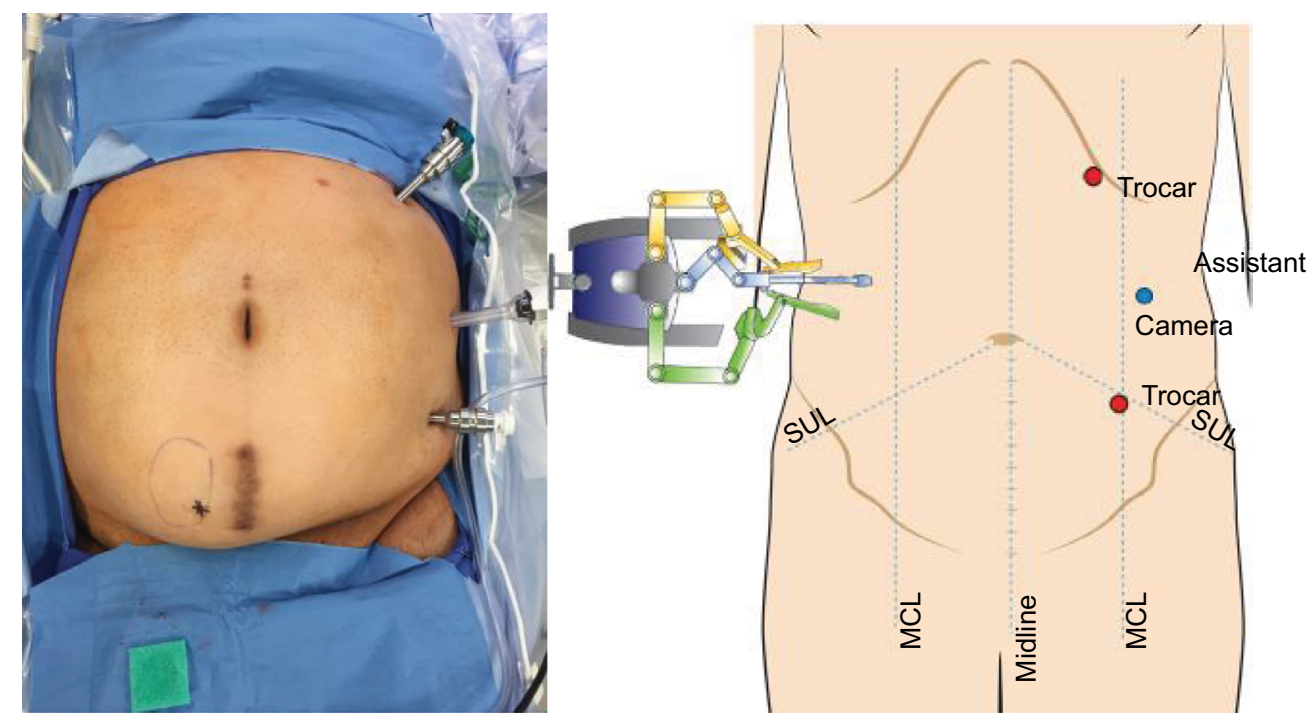

Figure 7 Lateral placement of ports maximizes the work field.

Abbreviations: MCL, midclavicular line; SUL, spinoumbilical line. 


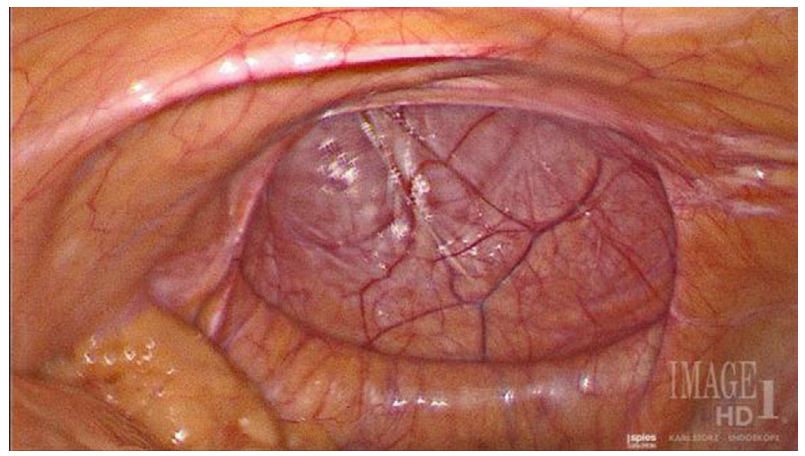

Figure 8 Large anterior incisional hernia defect.

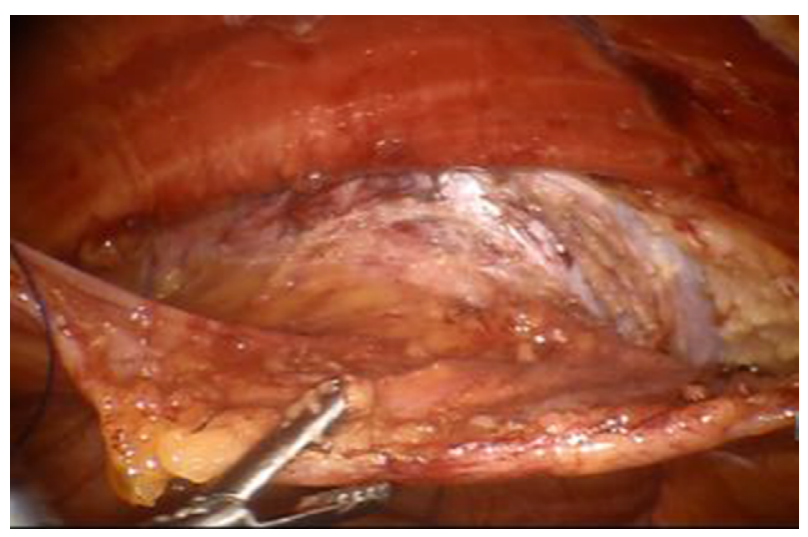

Figure 9 Retrorectus dissection of the sac.

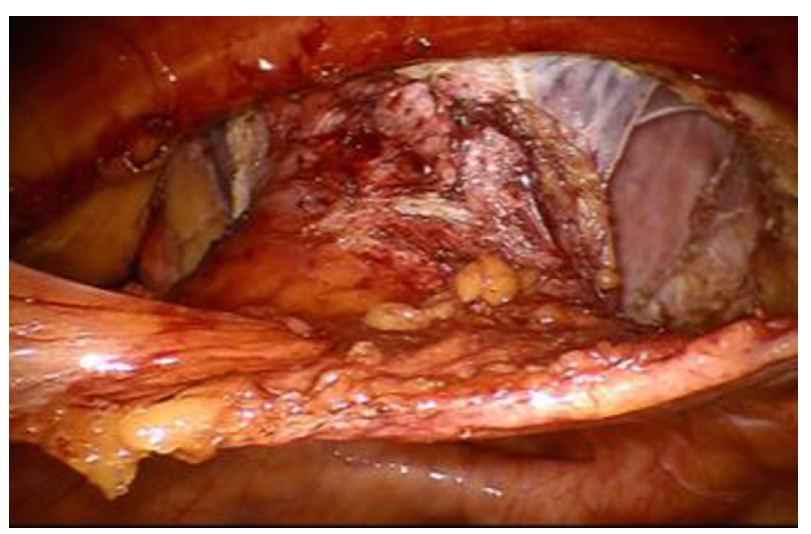

Figure 10 Complete circumferential dissection of the sac.

and incorporate it with the fascial closure. Once completely dissected, we approximate the fascial edges together with running barbed suture (Figure 11). Two sutures are used beginning at opposite ends. In cases where the defect may be too large to approximate the fascia comfortably, the transversus abdominis muscle can be released with either the hot shears or the vessel sealer device. The transversus abdominis muscle should be released just medial to the lateral perforating vessels, which become visible once the retromuscular dissection is taken to the semilunar line. The mesh is then placed against the rectus muscle that can be secured in place with tackers or

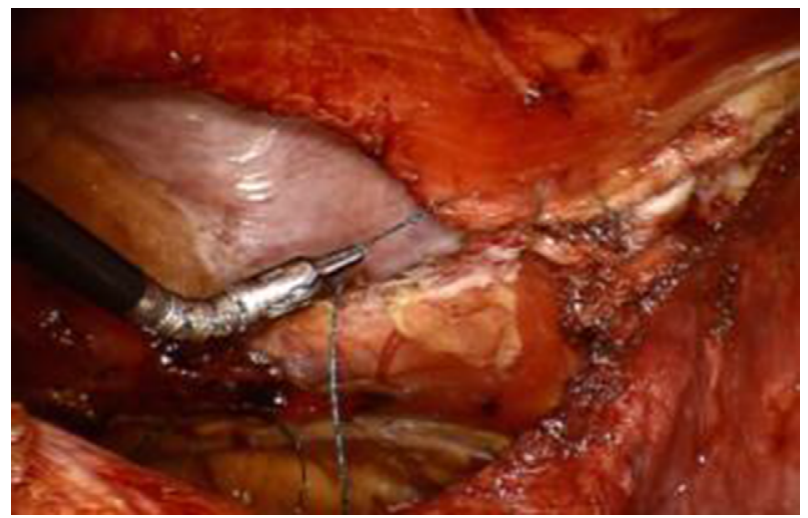

Figure I I Fascial edges are approximated with a running barbed suture.

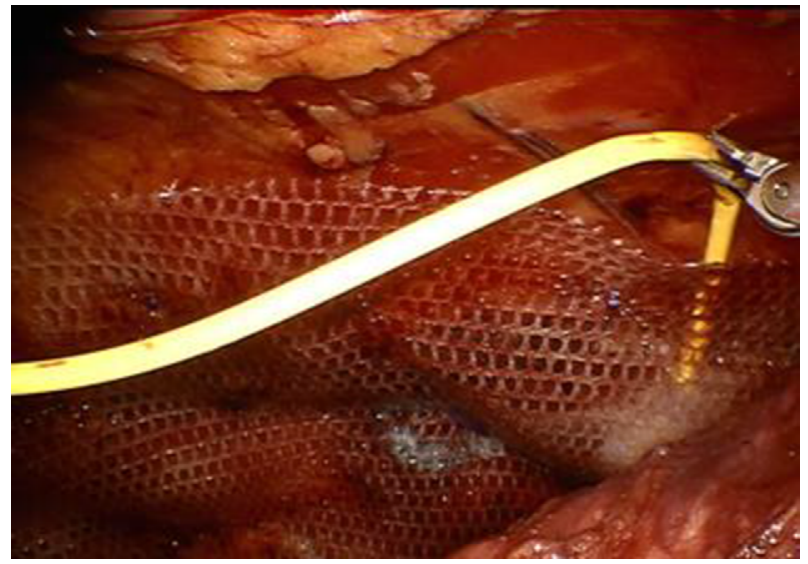

Figure 12 Tackers, transfixation suture, glue, or a combination is used to secure the mesh against the rectus muscle.

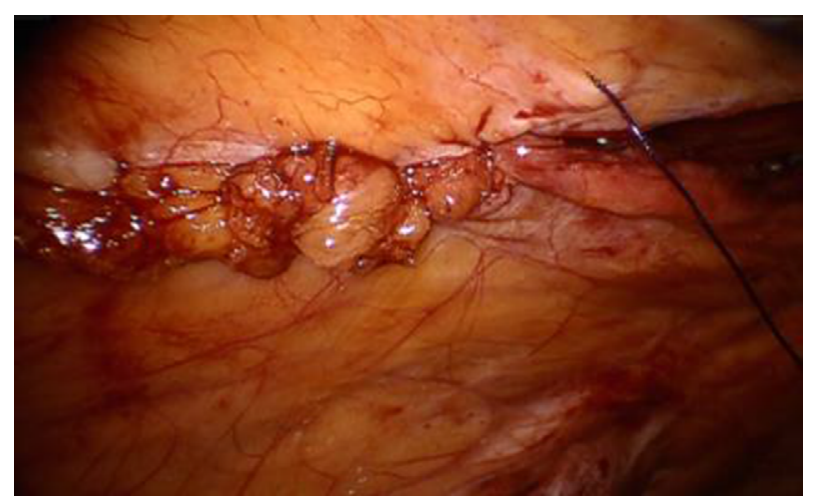

Figure 13 The posterior rectus sheath and peritoneum are closed securing the mesh in the retrorectus space and keeping it out of the intra-abdominal compartment.

transfixation sutures or glue (Figure 12). Self-adhering mesh is also an option. The posterior rectus sheath and peritoneum are closed over the mesh leaving none of it visible or in contact with intra-abdominal contents (Figure 13).

If the surgeon prefers the underlay or does not wish to place the mesh in the preperitoneal position, the technique mentioned earlier will vary. After access into the peritoneal cavity as previously described, all intra-abdominal adhesions are cleared and the sac is emptied. Removal of the sac can be 
performed also. Closure of the defect is accomplished with interrupted sutures or a running barbed suture. Mesh is subsequently placed over the repair with a minimum of $5 \mathrm{~cm}$ of overlap in all directions. Fixation of mesh can be completed with a running barbed suture circumferentially, interrupted sutures, tacks, or any of these combined.

Irrespective of the technique used, intraoperative placement of an abdominal binder with additional gauze rolls over the previous location of the hernia may help reduce the chance of a seroma or hematoma formation. This pressure dressing should be prescribed to the patient to be worn at all times for 1 month.

\section{Conclusion}

The robotic platform is proving to be a benefit to hernia repair. Many studies are showing its feasibility and comparable results to standard laparoscopy, and some have shown improved results including shorter hospital stay without significant cost increases. Complex abdominal wall reconstruction is a reasonable option for the experienced robotic hernia surgeon. Technical benefits include better visualization, improved ergonomics, and wristed movements allowing easier dissection. The robotic option of hernia repair has resulted in an increase in minimally invasive hernia repair, a number that has remained stagnant for the last decade. With greater availability of the robotic platform and more surgeons gaining experience and training, we expect to see greater numbers of minimally invasive hernia repair.

\section{Disclosure}

The authors report no conflicts of interest in this work.

\section{References}

1. Lichtenstein IL, Shulman AG. Ambulatory outpatient hernia surgery. Including a new concept, introducing tension-free repair. Int Surg. 1986;71(1):1-4.

2. Legutko J, Pach R, Solecki R, Matyja A, Kulig J. Rhys historyczny leczenia chirugicznego przepuklin [The history of treatment of groin hernia]. Folia Med Cracov. 2008;49(1-2):57-74. Polish.

3. McCormack K, Scott NW, Go PM, Ross S, Grant AM. Laparoscopic techniques versus open techniques for inguinal hernia repair. Cochrane Database Syst Rev. 2003;1:CD001785.

4. Tsui C, Klein R, Garabrant M. Minimally invasive surgery: national trends in adoption and future directions for hospital strategy. Surg Endosc. 2013;27(7):2253-2257.

5. Brown CN, Finch JG. Which mesh for hernia repair? Ann R Coll Surg Engl. 2010;92(4):272-278.

6. Patel PP, Love MW, Ewing JA, Warren JA, Cobb WS, Carbonell AM. Risks of subsequent abdominal operations after laparoscopic ventral hernia repair. Surg Endosc. Epub 2016 Jun 23.

7. Sajid MS, Ladwa N, Kalra L, McFall M, Baig MK, Sains P. A meta-analysis examining the use of tacker mesh fixation versus glue mesh fixation in laparoscopic inguinal hernia repair. Am J Surg. 2013;206(1):103-111.

8. Karigoudar A, Gupta AK, Mukharjee S, Gupta N, Durga CK. A prospective randomized study comparing fibrin glue versus prolene suture for mesh fixation in Lichtenstein inguinal hernia repair. Indian J Surg. 2016;78(4):288-292.
9. Shi Z, Fan X, Zhai S, Zhong X, Huang D. Fibrin glue versus staple for mesh fixation in laparoscopic transabdominal preperitoneal repair of inguinal hernia: a meta-analysis and systematic review. Surg Endosc. Epub 2016 Jun 28.

10. Morales-Conde S, Cadet H, Cano A, Bustos M, Martin J, MoralesMendez S. Laparoscopic ventral hernia repair without sutures - double crown technique: our experience after 140 cases with a mean follow-up of 40 months. Int Surg. 2005;90(3 suppl):S56-S62.

11. Gonzalez AM, Romero RJ, Seetharamaiah R, Gallas M, Lamoureux J, Rabaza JR. Laparoscopic ventral hernia repair with primary closure versus no primary closure of the defect: potential benefits of the robotic technology. Int J Med Robot. 2015;11(2):120-125.

12. Tabbara M, Genser L, Bossi M, et al. Inguinal hernia repair using selfadhering sutureless mesh: adhesix: a 3-year follow-up with low chronic pain and recurrence rate. Am Surg. 2016;82(2):112-116.

13. Negro P, Gossetti F, Ceci F, D'Amore L. Made in Italy for hernia: the Italian history of groin hernia repair. Ann Ital Chir. 2016;87:118-128.

14. Escobar Dominguez JE, Gonzalez A, Donkor C. Robotic inguinal hernia repair. J Surg Oncol. 2015;112(3):310-314.

15. Reynolds W Jr. The first laparoscopic cholecystectomy. JSLS. 2001;5(1):89-94.

16. Mathur S, Lin SY. The learning curve for laparoscopic inguinal hernia repair: a newly qualified surgeon perspective. J Surg Res. 2016; 205(1):246-251.

17. Finley DS, Rodriguez E Jr, Ahlering TE. Combined inguinal hernia repair with prosthetic mesh during transperitoneal robot assisted laparoscopic radical prostatectomy: a 4-year experience. $J$ Urol. 2007;178(4 pt 1): 1296-1299; discussion 1299-1300.

18. Joshi AR, Spivak J, Rubach E, Goldberg G, DeNoto G. Concurrent robotic trans-abdominal pre-peritoneal (TAP) herniorrhaphy during robotic-assisted radical prostatectomy. Int $J$ Med Robot. 2010;6(3):311-314.

19. Escobar Dominguez JE, Ramos MG, Seetharamaiah R, Donkor C, Rabaza J, Gonzalez A. Feasibility of robotic inguinal hernia repair, a single-institution experience. Surg Endosc. 2016;30(9):4042-4048.

20. Waite KE, Herman MA, Doyle PJ. Comparison of robotic versus laparoscopic transabdominal preperitoneal (TAPP) inguinal hernia repair. J Robot Surg. 2016;10(3):239-244.

21. Segura-Sampedro JJ, Ashrafian H, Navarro-Sanchez A, Jenkins JT, Morales-Conde S, Martinez-Isla A. Small bowel obstruction due to laparoscopic barbed sutures: an unknown complication? Rev Esp Enferm Dig. 2015;107(11):677-680.

22. Sauerland S, Walgenbach M, Habermalz B, Seiler CM, Miserez M. Laparoscopic versus open surgical techniques for ventral or incisional hernia repair. Cochrane Database Syst Rev. 2011;3:CD007781.

23. Earle DB, Mark LA. Prosthetic material in inguinal hernia repair: how do I choose? Surg Clin North Am. 2008;88(1):179-201,x.

24. den Hartog D, Dur AH, Tuinebreijer WE, Kreis RW. Open surgical procedures for incisional hernias. Cochrane Database Syst Rev. 2008;3:CD006438.

25. Booth JH, Garvey PB, Baumann DP, et al. Primary fascial closure with mesh reinforcement is superior to bridged mesh repair for abdominal wall reconstruction. J Am Coll Surg. 2013;217(6):999-1009.

26. Stoikes N, Webb D, Powell B, Voeller G. Preliminary report of a sutureless onlay technique for incisional hernia repair using fibrin glue alone for mesh fixation. Am Surg. 2013;79(11):1177-1180.

27. Rives J, Pire JC, Flament JB, Convers G. Le traitement des grandes éventrations. (A propos de 133 cas.) [Treatment of large eventrations (apropos of 133 cases)]. Minerva chirurgica. 1977;32(11):749-756. French.

28. Holihan JL, Bondre I, Askenasy EP, et al. Sublay versus underlay in open ventral hernia repair. J Surg Res. 2016;202(1):26-32.

29. Köhler L, Sauerland S, Meyer A, et al. Mesh implantation in onlay- or sublay- technique for closure of median ventral hernias: first results of a randomized clinical trial [Netzimplantation in Onlay-oder SublayTechnik zum Verschluss medianer Bauchwandhernien: erste Ergebnisse einer randomisierten klinishen Studie]. Poster presented at the Congress of the German Surgical Association; Berlin, Germany; May 5, 2006. 
30. Muysoms F, Campanelli G, Champault GG, et al. EuraHS: the development of an international online platform for registration and outcome measurement of ventral abdominal wall hernia repair. Hernia. 2012; $16(3): 239-250$.

31. LeBlanc KA, Booth WV. Laparoscopic repair of incisional abdominal hernias using expanded polytetrafluoroethylene: preliminary findings. Surg Laparosc Endosc. 1993;3(1):39-41.

32. Ramshaw BJ, Esartia P, Schwab J, et al. Comparison of laparoscopic and open ventral herniorrhaphy. Am Surg. 1999;65(9):827-831; discussion 831-822.

33. Liang MK, Clapp M, Li LT, Berger RL, Hicks SC, Awad S. Patient Satisfaction, chronic pain, and functional status following laparoscopic ventral hernia repair. World J Surg. 2013;37(3):530-537.
34. Halm JA, de Wall LL, Steyerberg EW, Jeekel J, Lange JF. Intraperitoneal polypropylene mesh hernia repair complicates subsequent abdominal surgery. World J Surg. 2007;31(2):423-429; discussion 430 .

35. Allison N, Tieu K, Snyder B, Pigazzi A, Wilson E. Technical feasibility of robot-assisted ventral hernia repair. World J Surg. 2012;36(2): $447-452$.

36. Warren JA, Cobb WS, Ewing JA, Carbonell AM. Standard laparoscopic versus robotic retromuscular ventral hernia repair. Surg Endosc. 2017;31(1):324-332.

37. Liang MK, Holihan JL, Itani $\mathrm{K}$, et al. Ventral hernia management: expert consensus guided by systematic review. Ann Surg. Epub 2016 Mar 15.
Robotic Surgery: Research and Reviews

\section{Publish your work in this journal}

Robotic Surgery: Research and Reviews is an international, peer reviewed, open access, online journal publishing original research, commentaries, reports, and reviews on the theory, use and application of robotics in surgical interventions. Articles on the use of supervisory-controlled robotic systems, telesurgical devices, and shared-control systems are

\section{Dovepress}

invited. The manuscript management system is completely online and includes a very quick and fair peer review system, which is all easy to use. Visit http://www.dovepress.com/testimonials.php to read real quotes from published authors. 